\title{
Disrupted-in-schizophrenia 1 enhances the quality of circadian rhythm by stabilizing BMAL1
}

\author{
Su Been Lee $\mathbb{D}^{1}$, Jihyun Park', Yongdo Kwak ${ }^{1,3}$, Young-Un Park ${ }^{1,4}$, Truong Thi My Nhung ${ }^{1}$, Bo Kyoung Suh', \\ Youngsik Woo (1)', Yeongjun Suh', Eunbyul Cho', Sehyung Cho² and Sang Ki Park (1)
}

\begin{abstract}
Disrupted-in-schizophrenia 1 (DISC1) is a scaffold protein that has been implicated in multiple mental disorders. DISC1 is known to regulate neuronal proliferation, signaling, and intracellular calcium homeostasis, as well as neurodevelopment. Although DISC1 was linked to sleep-associated behaviors, whether DISC1 functions in the circadian rhythm has not been determined yet. In this work, we revealed that Discl expression exhibits daily oscillating pattern and is regulated by binding of circadian locomotor output cycles kaput (CLOCK) and Brain and muscle Arntlike protein-1 (BMAL1) heterodimer to E-box sequences in its promoter. Interestingly, Discl deficiency increases the ubiquitination of BMAL1 and de-stabilizes it, thereby reducing its protein levels. DISC1 inhibits the activity of GSK3 $\beta$, which promotes BMAL1 ubiquitination, suggesting that DISC1 regulates BMAL1 stability by inhibiting its ubiquitination. Moreover, Disc1-deficient cells and mice show reduced expression of other circadian genes. Finally, Disc1-LI (Disc1 knockout) mice exhibit damped circadian physiology and behaviors. Collectively, these findings demonstrate that the oscillation of DISC1 expression is under the control of CLOCK and BMAL1, and that DISC1 contributes to the core circadian system by regulating BMAL1 stability.
\end{abstract}

\section{Introduction}

Anticipating the time of day is crucial for organisms that live under recurrent sunlight. The molecular circadian clock system maintains the daily cycle of organisms ${ }^{1-5}$. Transcription-translation feedback loop (TTFL) of circadian genes ensures this cycle, which keeps the circadian clock ticking even without external time cues ${ }^{5}$. In the mammalian TTFL system, the main component is the heterodimer consisting of Circadian locomotor output cycles kaput (CLOCK) and Brain and muscle Arnt-like protein-1 (BMAL1), which binds to E-box sequences of target genes for transcriptional enhancement ${ }^{6,7}$. In this way, the CLOCK/BMAL1 heterodimer upregulates core clock genes, including Period (PERs), Cryptochrome

\footnotetext{
Correspondence: Sehyung Cho (sehyung@khu.ac.kr) or

Sang Ki Park (skpark@postech.ac.kr)

'Department of Life Sciences, Pohang University of Science and Technology, Pohang, Republic of Korea

${ }^{2}$ Department of Physiology, College of Medicine, Kyung Hee University, Seoul, Republic of Korea

Full list of author information is available at the end of the article
}

$(C R Y S)$, and Nuclear receptor subfamily $1(N r 1 d 1)^{5}$. In turn, the action of CLOCK/BMAL1 heterodimer is negatively regulated by PERs and CRYs, thus completing the TTFL $^{8,9}$.

Timed regulation of circadian proteins is essential to the proper working of the circadian timing system. To this end, the degradation of the molecular circadian components is vital. Newly synthesized circadian proteins must be degraded at the right time for the accurate pacing of the circadian clock. Indeed, several circadian components, including BMAL1, PERs, CRYs, and REV-ERBs, are regulated by degradation pathways ${ }^{10}$. Glycogen synthase kinase $3 \beta$ (GSK3 $\beta$ ) phosphorylates BMAL1, thereby enhancing its ubiquitination and degradation ${ }^{11}$. Moreover, casein kinases $I \delta / \varepsilon(C K I \delta / \varepsilon)$ phosphorylate PERs leading to their degradation ${ }^{10}$. Chemical inhibition of CKI augments the stability of PERs and induces a more extended circadian period. F-box and leucine-rich repeat protein 3 (FBXL3), an E3 ubiquitin ligase, mediates ubiquitination and subsequent degradation of $\mathrm{CRYs}^{12}$, 
and FBXL3-mutated mice also exhibit longer circadian period $^{13}$. REV-ERB $\alpha$ is phosphorylated by cyclindependent kinase 1 (CDK1) and recognized for ubiquitination by F-box and WD repeat domain containing 7 $\left(\right.$ FBXW7) ${ }^{14}$. The loss-of-function of FBXW7 results in a damped circadian amplitude, indicating that the degradation of REV-ERB $\alpha$ is essential for the circadian amplitude. Altogether, these studies indicate that proper degradation of circadian proteins is a critical factor to maintain circadian period and amplitude.

Disrupted-in-schizophrenia 1 (DISC1) was first reported as a responsible gene for prevalent psychiatric conditions, including schizophrenia, in a Scottish pedigree $^{15}$. Although DISC1 as a genetic risk factor for schizophrenia has more to be elucidated, DISC1 plays significant molecular roles, including functions in early brain development, which are important to the molecular basis of psychiatric disorders ${ }^{16}$. DISC1 is a scaffold protein with a large number of interacting partners that perform various functions in the nervous system, such as neuronal migration, neurite outgrowth, spine regulation, and synapse maintenance ${ }^{17-20}$. Interestingly, DISC1 is associated with sleep-related phenotypes. Expression of human DISC1 in fruit flies alters their sleep homeostasis $^{21}$. Furthermore, a mouse model of DISC1 gain-offunction shows increased wakefulness and decreased REM and NREM sleep ${ }^{22}$. A close relationship between sleep and the circadian clock has been proposed ${ }^{23}$; however, potential direct involvement of DISC1 in the mammalian circadian system has not been explored yet. In this study, we investigated the relationship between DISC1 and the circadian system and proposed a modulatory role of DISC1 in the mammalian molecular clock.

\section{Materials and methods Animals}

All animal-related experiments were approved by Pohang University of Science and Technology Institutional Animal Care and Use Committee (POSTECH2019-0024 and POSTECH-2019-0025), and animal experiments were conducted with approved guidelines. Wild type C57BL/6J mice were purchased from Hyochang Science (Daegu, South Korea). Disc1 knockout mouse, named as Disc1 locus impairment mouse (Disc1-LI mouse, C57BL/6 J backgrounded), was previously described $^{24}$ and a kind gift from Dr. Akira Sawa, Johns Hopkins University School of Medicine. In Disc1-LI mouse, exons from 1 to 3 of Disc1 were replaced to Neomycin resistance cassette by homologous recombination ${ }^{24,25}$. Male wild type (C57BL/6J) mice and Disc1-LI mice were housed under 12-h light 12-h dark conditions with ad libitum access to food and water. Male mice aged 3 to 4 months were employed for circadian experiments.

\section{Cell culture and transfection}

HEK293 cell line, NIH3T3 cell line, and Mouse embryonic fibroblasts (MEFs) were maintained in $5 \% \mathrm{CO}_{2}$ incubator at $37^{\circ} \mathrm{C}$ with DMEM high glucose (Welgene, LM 001-05) containing 10\% fetal bovine serum (Merck, ES009B-KC) and 1\% penicillin/streptomycin (HyClone, SV30010). Cells were transfected with Polyethylenimine (PEI, Polysciences, Inc., 23966-2) as previously described $^{26}$. Briefly, plasmids were mixed with PEI $(1 \mathrm{mg} / \mathrm{ml}$, $\mathrm{pH}$ 7.0) in opti-MEM (Gibco, 31985-070) and added to cells after 15 min of incubation.

\section{Antibodies}

For antibodies, rabbit anti-DISC1 (Millipore, ABN-425) was purchased from Millipore; rabbit anti-BMAL1 (Abcam, ab93806) from Abcam; mouse anti-alphatubulin (Proteintech, 66031-1-lg) from Proteintech; rabbit anti-HA (Bethyl, A190-108A) from Bethyl; rabbit anti-GFP (Invitrogen, A11122) from Invitrogen; rabbit anti-Flag (ThermoFisher, PA1-984B) from ThermoFisher; mouse anti-Flag (Sigma, F1804) from Sigma; mouse antic-Myc (Santa Cruz, sc-40) from Santa Cruz; GSK3 $\beta$ p-Y216 (Invitrogen, 44-604G) from Invitrogen; mouse anti-GSK3 $\beta$ (Cell Signaling, 9832S) from Cell Signaling. Antibodies were utilized as the first antibody of western blot or precipitating antibody of immunoprecipitation.

\section{Plasmids}

Human DISC1 promoter ( -982 to +47 relative to $\mathrm{TSS}^{27}$ ) was cloned in pGL3-basic (Promega), whose luciferase was modified as destabilized with PEST sequence ${ }^{28}$. For distal, middle, and proximal promoter regions of DISC1, -982 to $-624,-623$ to -324 , and -323 to +47 relative to TSS respectively, were cloned in pGL3-basic with destabilized luciferase. PCR and DpnI-based mutation method was utilized to mutate E-box sequences of DISC1 promoter. Primers were used for the PCR; for mutant E-box ( -718 relative to TSS): 5'-TCTATGACCGTACTC TCCTTC- $3^{\prime}$ and $5^{\prime}$-GAAGGAGAGTACGGTCATAGA3'; for mutant E-box ( -668 relative to TSS): 5'-AGAGC TACTGTATAGCCCTTC- $3^{\prime}$ and $5^{\prime}$-GAAGGGCTATA CAGTAGCTCT- ${ }^{\prime}$. Human CLOCK cDNA was cloned in mRFP-C1 with myc tagging at C-terminal. Human BMAL1 cDNA was cloned in pEGFP-C1 (Clontech) and pcDNA3.1 myc-His C (Invitrogen). HA-hDISC1 was a kind gift from Dr. Akira Sawa, Johns Hopkins University School of Medicine, and sub-cloned in pFLAG-CMV2 (Sigma). Human GSK3 $\beta$ cDNA was cloned in pcDNA3.1 myc-His $\mathrm{C}$ (Invitrogen). HA-Ubiquitin was a kind gift from Dr. Chin Ha Chung, Seoul National University, Seoul, Korea. pLL3.7 vector was utilized for shRNA knockdown experiments. Target sequence for control shRNA, 5'-ACTACCGTTGTATAGGTG-3', was previously described ${ }^{29}$; $5^{\prime}$-GCAGGAGGTCAGCAAGGCC 
TTG-3' for human DISC1 shRNA was previously described $^{30}$.

\section{Western blot}

For western blot, cells or brain samples were lysed in Nonidet P-40 buffer (50 mM Tris-Cl, pH 8.0, $150 \mathrm{mM}$ $\mathrm{NaCl}, 1 \%$ NP40, $5 \mathrm{mM}$ EDTA, 1X protease inhibitor cocktail). Samples were sonicated and centrifuged at $13,000 \mathrm{rpm}, 4^{\circ} \mathrm{C}$ for $10 \mathrm{~min}$ to remove debris. Acquired supernatant was mixed with SDS sampling buffer and denatured at $95^{\circ} \mathrm{C}$ for $10 \mathrm{~min}$. Denatured samples were separated with SDS-PAGE gel electrophoresis and transferred to PVDF membrane (Immobilon-PSQ PVDF Membrane, Millipore, ISEQ00010). Membranes were blocked with $5 \%$ skim milk and incubated with each appropriate first antibody for overnight at $4{ }^{\circ} \mathrm{C}$, then washed 3 times with TBS-T buffer $(20 \mathrm{mM}$ Tris- $\mathrm{Cl}, \mathrm{pH}$ 7.6, $137.5 \mathrm{mM} \mathrm{NaCl}, 0.25 \%$ Tween 20). Further, membranes were incubated with Horseradish peroxidase (HRP) conjugated second antibody for $1 \mathrm{~h}$, followed by 3 times washing with TBS-T buffer. ECL solution (Bio-rad, 1705061) was applied to membranes, and the band signals were detected by chemiluminescent detector (Azure, c280).

\section{Quantitative RT-PCR}

Tri-solution (Bio Science Technology, TS200-001) was used for extracting RNA from cells or brain samples. The RNA concentration was measured by Nanodrop 2000 (Thermo), and $1 \mu \mathrm{g}$ of RNA was subjected to further experiments. ImProm-II ${ }^{\mathrm{TM}}$ Reverse Transcription System (Promega, A3800) and poly $\mathrm{T}$ primer were used for reverse transcript of RNA samples. FastStart Universal SYBR Green Master (Rox) (Roche, 04913850001) and StepOnePlus Real-Time PCR System (Applied Biosystems) were utilized to conduct qPCR according to manufacturer's instructions with primers for each gene:

Period1 F: 5'-GCCAAGAAAGATCCGTCGTCAG-3'

Period1 R: 5'-GGGCTTCTTGTCTCCCACATGGAC$3^{\prime}$ (ref. $^{31}$ )

Period2 F: 5'-GCTTCTGGTCTGGACTGCAC-3'

Period2 R: $5^{\prime}$-GAGTGTCTGAGGGCTCGTTG-3' (ref. ${ }^{32}$ )

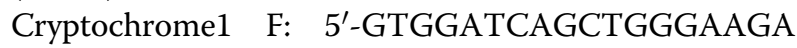
AG-3'

Cryptochrome1 R: 5'-CACAGGGCAGTAGCAGTGA A-3' (ref. ${ }^{33}$ )

Bmal1 F: 5'-GCAGTGCCACTGACTACCAAGA-3'

Bmal1 R: $5^{\prime}$-TCCTGGACATTGCATTGCAT- $3^{\prime}$ (ref. ${ }^{34}$ )

Disc1 F: 5'-GAACAGCAGAAGGCTGGGC-3'

Disc1 R: 5'-GACCTTCCAACACTTCCATGC-3' (ref. $^{35}$ )

Tbp F: 5'-GGGAGAATCATGGACCAGAA- $3^{\prime}$

Tbp R: $5^{\prime}$-CCGTAAGGCATCATTGGACT- $3^{\prime}$ (ref. ${ }^{36}$ ) Gapdh F: 5'-CACTGAAGGGCATCTTGG-3'
Gapdh R: $5^{\prime}$-TTACTCCTTGGAGGCCATG-3' (ref. ${ }^{37}$ ) Gapdh or TATA-Box Binding Protein (TBP) was utilized as a reference gene for qRT-PCR according to previous studies ${ }^{38-42}$.

\section{Mouse embryonic fibroblasts}

Mouse embryonic fibroblasts (MEFs) were obtained from wild type C57BL/6J mouse and Disc1-LI C57BL/6J mouse line. Embryos with embryonic day 14.5 were used. Body part of embryos without red blood organs was washed with PBS (Welgene, ML 008-01) and minced with blades. Samples were dissociated further with trypsinEDTA (HyClone, SH30042.02) and pipetting for $10 \mathrm{~min}$. Complete media (DMEM with 10\% FBS and $1 \%$ penicillin/streptomycin) was applied to block trypsin activity. Collected cells were centrifuged for $3 \mathrm{~min}$ at $1000 \mathrm{rpm}$ and pellets were resuspended with complete media. To dissociate into single cells, cell strainer was applied. Dissociated cells were seeded into culture dishes and media change was conducted after $6 \mathrm{~h}$.

\section{Circadian rhythm experiments}

For cultured cell experiments, $1 \mu \mathrm{M}$ of dexamethasone (Sigma, D4902-25MG) was applied to synchronize cells' circadian phase. After $2 \mathrm{~h}$, cells were changed to fresh complete media (DMEM with 10\% FBS and 1\% penicillin/ streptomycin) and were harvested according to each time point. To obtain mouse brain samples for circadian experiments, mice were entrained to a 12-h light and 12-h dark cycle for 7 days and then to constant darkness to monitor internal circadian phenotypes. After the constant dark cycle had started, mice were kept for 1 day and sacrificed in the next day. $36 \mathrm{~h}$ after the constant dark cycle had started was considered circadian time 0 . Mice were sacrificed under dim red light according to their circadian times and further dissection for brains was performed.

For long-term monitoring of circadian behavior in live animals, mice were surgically implanted with telemetric proves (E-mitter; Mini-Mitter, Bend) and kept in a lightproof Clean Animal Rack cabinet (Shin Biotech) equipped with an automatic light-dark schedule controller. The light intensity during the light phase was kept at 350-450 lux all over the cabinet. The temperature inside the cabinet was maintained to $23 \pm 1{ }^{\circ} \mathrm{C}$, and constant air ventilation was applied all the time. Prior to the actual monitoring, mice were fully entrained to a 12 -h light and 12-h dark cycle for at least two weeks. For the next 10 days with the same light-dark cycle, wheel-running activity, body temperature, and home cage activity were continuously recorded at 6-min intervals using ER-4000 system. Data obtained during this period were used for the construction of light-dark profiles of behaviors. Then, mice were released to constant darkness (dark-dark cycle) 
for 10 days to determine the free-running period of circadian behaviors. For phase delay assay or phase advance assay, 30 min of light pulse was given to mice on circadian time $14 \mathrm{~h}$ or $20 \mathrm{~h}$, respectively. Thereafter, the phase change was measured.

\section{Luciferase assay}

Luciferase assay was conducted using Dual Luciferase Assay system (Promega, E1910), according to manufacturer's instruction. Cells were cultured on 12-well plate and transfected with PEI. Prior to cell prep, cells were washed with PBS (Welgene, ML 008-01). $100 \mu$ l of passive lysis buffer (PLB) was applied to each well, and the plate was agitated for $10 \mathrm{~min}$ to detach cells from the plate. The lysate was collected and centrifuged for $30 \mathrm{~s}$ at $13,000 \mathrm{rpm}$. The $40 \mu \mathrm{l}$ of supernatant was transferred to 96-well white plate (Nunc, 136101). $50 \mu \mathrm{l}$ of luciferase assay buffer II was injected into a well of 96-well plate, and shaking was performed for 1 second. Thereafter, Firefly luciferase activity was measured for $10 \mathrm{~s} .50 \mu \mathrm{l}$ of Stop \& Glo Buffer was injected into the same well of 96well plate, and shaking was performed for $1 \mathrm{~s}$. Thereafter, Renilla luciferase activity was measured for $10 \mathrm{~s}$. Injection of substrates and luminescence detection were programmed and conducted by automatic injector and plate reader (TECAN, infinite M200 PRO).

\section{Immunoprecipitation and ubiquitination assay}

Cultured cells, transfected with proper plasmids for each experiment, were lysed with Nonidet P-40 buffer (50 mM Tris-Cl, pH 8.0, $150 \mathrm{mM} \mathrm{NaCl}, 1 \% \mathrm{NP} 40,5 \mathrm{mM}$ EDTA, $1 \times$ protease inhibitor cocktail). Brain samples were homogenized in Nonidet P-40 buffer. Samples were sonicated and centrifuged at $13,000 \mathrm{rpm}, 4^{\circ} \mathrm{C}$ for $10 \mathrm{~min}$. The supernatants were incubated with $500 \mathrm{ng}$ of antibodies via rotation at $4{ }^{\circ} \mathrm{C}$. Protein A beads (Roche, 05015979001) were washed three times with Nonidet P-40 buffer. Antibody incubated samples were added to washed beads and incubated further for $4 \mathrm{~h}$ at $4{ }^{\circ} \mathrm{C}$ with rotation. Beads, with bound proteins, were precipitated with $1 \mathrm{~min}$ of centrifugation at $2000 \mathrm{rpm}, 4^{\circ} \mathrm{C}$. Beads were washed three times with Nonidet P-40 buffer and denatured with SDS sampling buffer at $95^{\circ} \mathrm{C}$ for $10 \mathrm{~min}$. Samples were further subjected to immunoblotting. For ubiquitination assay, cells had been transfected with plasmids, including HA-ubiquitin; thereafter, $10 \mathrm{mM}$ of MG132 (Sigma, C2211-5MG) was treated for $5 \mathrm{~h}$ prior to the cell lysis. Further experimental procedure is identical to immunoprecipitation assay.

\section{Chromatin immunoprecipitation}

Cultured cells were transfected with appropriate plasmids. Cells were treated with $0.75 \%$ of formaldehyde (Sigma, 252549) dropwise and incubated for $10 \mathrm{~min}$ with slow agitation. $125 \mathrm{mM}$ of glycine was treated to stop cross-linking. After twice of PBS washing, cells were harvested with PBS and centrifuged for $5 \mathrm{~min}, 1000 \times g$, $4{ }^{\circ} \mathrm{C}$. Cells were resuspended with ChIP lysis buffer (50 mM HEPES-KOH pH 7.5, $140 \mathrm{mM} \mathrm{NaCl}, 1 \mathrm{mM}$ EDTA pH 8.0, 1\% Triton X-100, 0.1\% sodium deoxycholate, $0.1 \%$ SDS, $1 \times$ Protease Inhibitor) and incubated for $10 \mathrm{~min}$ on ice. Samples were sonicated for $20 \mathrm{~min}(30 \%$ amplitude, sonicate/rest for $3 \mathrm{~s} / 3 \mathrm{~s}$ ), and cell debris was removed with centrifuge for $10 \mathrm{~min}, 13,000 \mathrm{rpm}, 4^{\circ} \mathrm{C}$. Samples were diluted with mRIPA buffer $(150 \mathrm{mM} \mathrm{NaCl}$, $50 \mathrm{mM}$ Tris- $\mathrm{HCl} \mathrm{pH} 7.5,1 \% \mathrm{NP}-40,1 \%$ Triton X-100, $0.5 \%$ sodium deoxycholate, $5 \mathrm{mM}$ EDTA). $50 \mu \mathrm{l}$ of samples were kept as an input. $25 \mu \mathrm{g}$ of total DNA samples were used each. Samples were incubated with Protein A beads (Roche, 05015979001 ) for $4 \mathrm{~h}$ at $4{ }^{\circ} \mathrm{C}$ with rotation to pre-clear non-specific bindings. Beads were removed by centrifuge for $1 \mathrm{~min}, 2,000 \mathrm{rpm}, 4^{\circ} \mathrm{C}$. Antibody was treated to samples and incubated for $4 \mathrm{~h}$ at $4{ }^{\circ} \mathrm{C}$ with rotation. New Protein A beads were washed three times with mRIPA buffer, and beads were incubated with samples for overnight. Incubated beads were washed once with Low salt buffer $(0.1 \%$ SDS, $1 \%$ Triton X-100, 2 mM EDTA, $20 \mathrm{mM}$ Tris- $\mathrm{HCl} \mathrm{pH}$ 8.0, $150 \mathrm{mM} \mathrm{NaCl}$ ), once with High salt buffer (0.1\% SDS, 1\% Triton X-100, 2 mM EDTA, $20 \mathrm{mM}$ Tris- $\mathrm{HCl} \mathrm{pH} 8.0,500 \mathrm{mM} \mathrm{NaCl}$ ), once with $\mathrm{LiCl}$ buffer $(0.25 \mathrm{M} \mathrm{LiCl}, 1 \% \mathrm{NP}-40,1 \%$ sodium deoxycholate, $1 \mathrm{mM}$ EDTA, $10 \mathrm{mM}$ Tris- $\mathrm{HCl} \mathrm{pH}$ 8.0) by centrifuge for $1 \mathrm{~min}, 2000 \mathrm{rpm}, 4^{\circ} \mathrm{C}$. Bead-attached samples were eluted from beads by incubation with $120 \mu \mathrm{l}$ of Elution buffer (1\% SDS, $100 \mathrm{mM} \mathrm{NaHCO}{ }_{3}$ ) for $15 \mathrm{~min}$ at $30^{\circ} \mathrm{C}$. Beads were removed by centrifuge for $1 \mathrm{~min}, 2000 \mathrm{rpm} .4 .8 \mu \mathrm{l}$ of $5 \mathrm{M} \mathrm{NaCl}$ and $2 \mu \mathrm{l}$ of $10 \mathrm{mg} / \mathrm{ml}$ RNase A (Thermo, EN0531) were added to samples and incubated for overnight at $65^{\circ} \mathrm{C}$ with agitation. $4 \mu \mathrm{l}$ of $10 \mathrm{mg} / \mathrm{ml}$ Proteinase $\mathrm{K}$ (Sigma, P2308) was added and incubated for $1 \mathrm{~h}$ at $60^{\circ} \mathrm{C}$ with agitation. Further, DNA was acquired by PCR purification kit. For inputs, $50 \mu \mathrm{l}$ of each input was mixed with $70 \mu \mathrm{l}$ of Elution buffer and followed the same procedure with bead samples. Promoter targets were quantified with qPCR. For E-boxes of DISC1 promoter, $5^{\prime}$-G GTTTTGTGCCAAGCCTCTG-3' $/ 5^{\prime}$-AGCTCACCTCC AGGCTAGAA- $3^{\prime}$ and $5^{\prime}$-GGAGGTGAGCTGCTTAAG GG-3'/5'-CCATGCAAGCTCCTAGGCAA-3' primer sets were utilized for qPCR.

\section{Quantification and statistical analysis}

Sample size was chosen based on previous studies. No statistical methods were used for sample size estimation. No randomization was used for animal studies. The investigators were not blinded to the group allocation during the animal experiments. Image $(\mathrm{NIH})$ was used for quantification of band intensity of western blot, and Prism 8 (GraphPad) was used for statistical analyses. 
Statistical significances were determined by unpaired $t$-test or paired $t$-test, two-tailed for comparisons of two groups. One-way ANOVA or two-way ANOVA was used for comparisons of multiple groups, followed by Tukey's multiple comparison test or Sidak's multiple comparison test, respectively. $P$-value less than 0.05 was considered a significant difference. If variances of the data were significantly different, we utilized a statistical test that does not assume homogenous variance; Welch's ANOVA, unpaired $t$-test with Welch's correction.

\section{Results}

DISC1 shows rhythmic expression relative to circadian time

To elucidate whether DISC1 is associated with the circadian system, we first examined whether DISC1 itself has an oscillatory expression pattern as other circadianrelated genes. Disc1 mRNA expression was assessed in NIH3T3 cells after synchronization induced by dexamethasone treatment. The mRNA level of Disc1 showed an oscillatory pattern that peaked at 24 and $40 \mathrm{~h}$ after synchronization (Fig. 1a). Subsequently, we examined this expression pattern on an organism level. We entrained mice to a 12-h light and 12-h dark cycle for a week, and then switched to constant darkness and sacrificed mice according to their circadian time. Remarkably, we revealed the oscillatory mRNA expression of Disc1 in the hippocampus (Fig. 1b). Disc1 mRNA peaked at circadian time $20 \mathrm{~h}$ (CT20), originally nighttime $(\sim 12-24 \mathrm{~h})$ for mice. The mRNA oscillation of Disc1 was also observed in the suprachiasmatic nucleus (SCN) (Fig. 1c). Disc1 mRNA peak was CT19. When compared to other circadian genes in SCN, Disc1 peak time is slower than Per1 and Per2, and faster than Rev-erbo ${ }^{43,44}$. Moreover, DISC1 endogenous protein levels also oscillated (Fig. 1d, e). Taken together, Disc1 expression shows an oscillatory pattern, with the expression level enhanced during late nighttime to early daytime.

\section{CLOCK and BMAL1 heterodimer regulates DISC1 promoter}

Next, we attempted to discover the factors that contribute to the oscillatory expression of DISC1 within the molecular circadian system. As shown in Fig. 1b, the mRNA expression of Disc1 increases in late nighttime and a little later, roughly similar to the expression pattern of Cry1. Since the expression of circadian genes, such as Cry1, Cry2, and Per2, is controlled by CLOCK/BMAL1 heterodimer ${ }^{5}$, we hypothesized that Disc1 expression could be regulated with a comparable mechanism.

Based on the previous characterization of DISC1 promoter $^{27}$, we further analyzed the region from -982 to +47 bp relative to the transcription start site (TSS). DISC1 promoter was cloned into pGL3 vector containing luciferase destabilized by PEST sequence ${ }^{28}$. We then assessed the activity of DISC1 promoter by luciferase assay system.
Interestingly, DISC1 promoter activity was enhanced when CLOCK and BMAL1 were co-expressed, but not when CLOCK was expressed alone (Fig. 2a). Next, fragments of the DISC1 promoter were sub-cloned into pGL3 vector. The distal part of DISC1 promoter (-982 to -624 bp relative to TSS) was highly enhanced by CLOCK and BMAL1 co-expression (Fig. 2b). The middle part of DISC1 promoter ( -623 to -324 bp relative to TSS) was also moderately enhanced by CLOCK and BMAL1 transfection, while the proximal part of DISC1 promoter $(-323$ to +47 bp relative to TSS) did not show significant enhancement. Therefore, the distal part of DISC1 promoter is the most responsive region to CLOCK/BMAL1.

To define the binding sites of CLOCK/BMAL1 heterodimer on DISC1 promoter, we utilized the 'LASAGNA-Search 2.0' tool $^{45,46}$. From the prediction based on the previously reported matrix parameter for CLOCK and BMAL1 ${ }^{47}$, two candidate binding sites $(-718$ and -668 bp relative to TSS) were identified (Supplementary Fig. 1). The two predicted sites match with the consensus E-box element, CANNTG, where CLOCK/ BMAL1 binds ${ }^{48-51}$. Then, the predicted E-box sequences of DISC1 promoter were mutated to 'ACNNTA' to block their activity. DISC1 promoter with mutations in either one of the two E-box sequences or both showed lower responses to CLOCK and BMAL1 overexpression than the wild type promoter (Fig. 2c). When the distal part of DISC1 promoter was tested separately, double mutation of E-box sequences abrogated the response to CLOCK and BMAL1 (Fig. 2d). Then, chromatin immunoprecipitation assay (ChIP) was employed to test whether CLOCK/BMAL1 heterodimer physically binds to the Ebox sequences of DISC1 promoter (Fig. 2e). In CLOCK/ BMAL1-cotransfected cells, qPCR signals for both Eboxes ( -718 and -668 bp relative to TSS) were markedly increased. In addition, we performed ChIP on mutant E-boxes ( -718 and -668 bp relative to TSS) of DISC1 promoter to examine the binding of CLOCK/BMAL1 heterodimer. The qPCR signals for both E-boxes were significantly decreased (Supplementary Fig. 2). Altogether, the results further support the idea that the two E-box sites within the distal part of DISC1 promoter are critical for the modulation of DISC1 expression by CLOCK/ BMAL1.

\section{DISC1 regulates BMAL1 stability and expression of circadian genes}

PERs and CRYs are regulated by CLOCK/BMAL1 through E-box sequences and exhibit oscillatory expression within circadian time ${ }^{5}$. Circadian expression of PERs and CRYs subsequently inhibits CLOCK/BMAL1 via a negative feedback loop. Since DISC1 expression is regulated by CLOCK/BMAL1 through E-box sequences and displays an oscillatory expression, we hypothesized that 


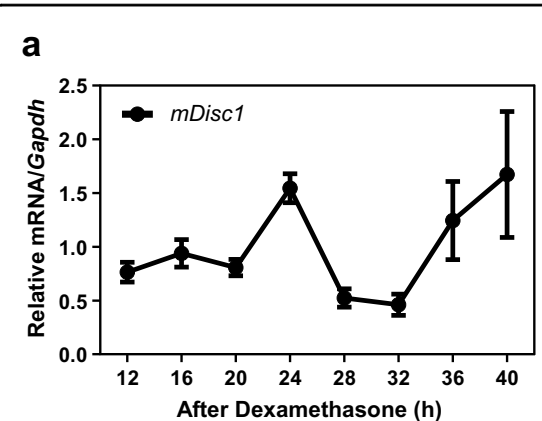

d

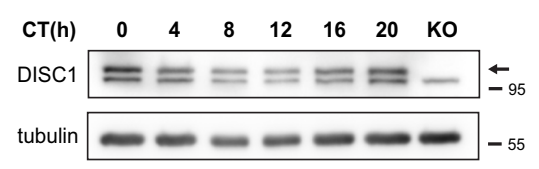

b

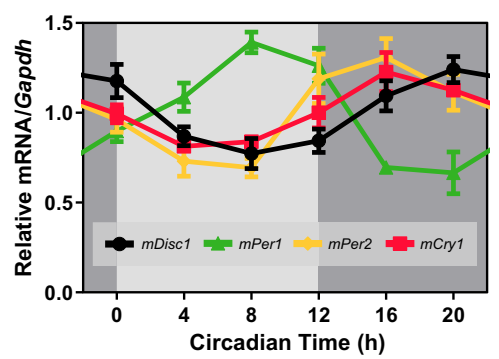

e

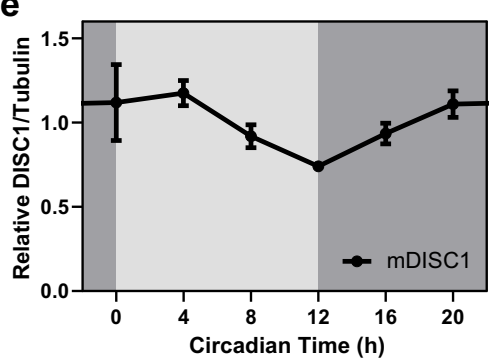

C

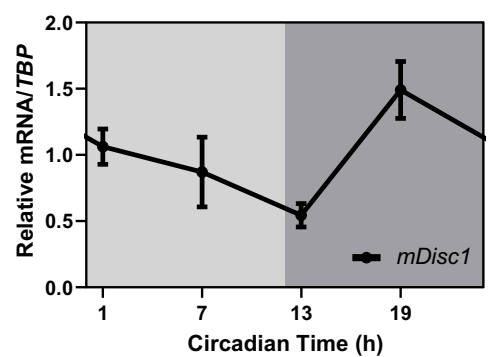

Fig. 1 Expression of DISC1 exhibits an oscillating pattern. a mRNA expression of Disc1 in NIH3T3 cells. Cells were treated with $1 \mu \mathrm{M}$ of dexamethasone for $2 \mathrm{~h}$ to synchronize the circadian cycle. mRNA levels of Discl were analyzed by qRT-PCR and compared to those of Gapdh ( $n=5$ for $12 \mathrm{~h}, n=6$ for other time points, biological replicates). The ratios of the relative mRNA levels at each time point to the mRNA levels averaged across all time points were presented. $p=0.0151$, one-way ANOVA. $\mathbf{b}$ mRNA level of Disc 1 in hippocampus of wild type mice $(n=3,3$ mice for each time point) during the circadian time. Mice were entrained on a 12-h light/12-h dark cycle for a week; thereafter, mice were in the constant dark for a day and were sacrificed under dim red light according to their circadian time. mRNA levels of Discl were analyzed using qRT-PCR and compared to those of Gapdh. To compare the expression patterns of circadian genes measured by qRT-PCR each other, the ratios of the relative mRNA levels at each time point to the mRNA levels averaged across all time points were presented. $p=0.0037$ for Discl, one-way ANOVA. c mRNA levels of Discl in suprachiasmatic nucleus (SCN) of wild type mice ( $n=3,3$ mice for $7 h ; n=4,4$ mice for other time points) during the circadian time. mRNA levels of Discl were analyzed by qRT-PCR and compared to those of TATA-box binding protein (TBP). The ratios of the relative mRNA levels at each time point to the mRNA levels averaged across all time points were presented. $p=0.0166$, one-way ANOVA. $\mathbf{d}$ DISC1 protein levels according to the circadian time in hippocampus were assessed by western blot. Wild type mice were entrained on a 12-h light/12-hr dark cycle for a week; thereafter, mice were in the constant dark condition and sacrificed under dim red light according to their circadian time. Disc1 knockout (Disc1-LI mouse) sample was used as a negative control. Arrow indicates DISC1 bands. e Quantification of DISC1 protein level from (d) ( $n=4$ for each time point, biological replicates). Protein levels were quantified relative to tubulin. The ratios of the relative band intensity at each time point to the band intensities averaged across all time points were presented. $p=0.0042$, Welch's ANOVA. Data are means with SEM.

DISC1 protein may also affect the molecular circadian system, and we scrutinized the potential links. Interestingly, we observed that ectopic expression of DISC1 enhanced BMAL1 protein level in HEK293 cells (Fig. 3a, b). Conversely, endogenous BMAL1 protein level was decreased in mouse embryonic fibroblasts (MEFs) derived from Disc1-LI mice, a Disc1-deficient mouse line $^{24}$ (Fig. 3c, d).

To verify whether DISC1 deficiency affects BMAL1 oscillation over circadian time, we analyzed BMAL1 protein expression at each circadian time after dexamethasone treatment (Fig. 3e, f). The circadian oscillation of BMAL1 was significantly damped in Disc1-LI MEFs, and this effect was higher when BMAL1 levels were at their peak, namely 20 and $24 \mathrm{~h}$ after dexamethasone treatment. Moreover, the mRNA levels of circadian genes under the control of BMAL1 were concomitantly reduced in Disc1-LI MEFs (Fig. 3g-j). Given that the knockout of
Disc1 damps BMAL1, thereby also decreasing the mRNA expression of Per2, Cry1, and Bmal1, these data strongly suggest that DISC1 may function as a stabilizer of BMAL1.

\section{Disc1 knockout mice display altered circadian behavior}

Since Disc1 knockout decreases the expression of circadian genes and decreases protein levels of BMAL1, we hypothesized that Disc1 knockout would also affect circadian behaviors. To test this hypothesis, we conducted circadian behavioral tests with Disc1-LI mice. We first entrained mice to a 12-h light and 12-h dark cycle prior to the observation of light/dark profile of circadian behavior. Notably, Disc1-LI mice exhibited lowered amplitude of circadian behaviors: wheel-running activity (Fig. 4a), body temperature (Fig. 4b), and home cage activity (Fig. 4c). We then monitored the wheel-running activity profile in a constant dark/dark cycle to observe the internal circadian 


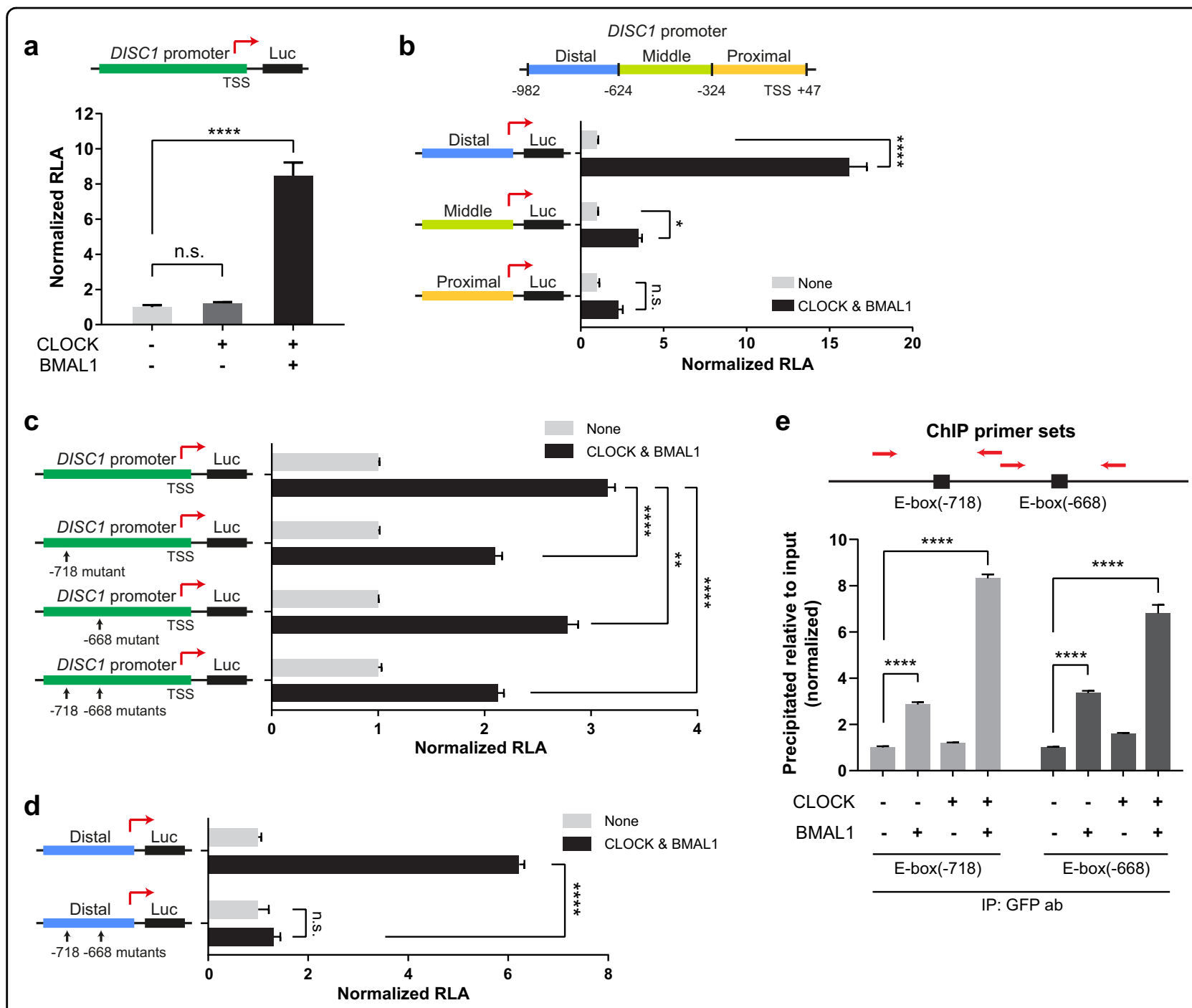

Fig. 2 Promoter activity of DISC1 is enhanced by CLOCK and BMAL1 through E-box sequences. a DISC1 promoter was cloned into pGL3 vector to monitor promoter activity by luciferase expression. Luciferase assay was performed in HEK293 cells overexpressing CLOCK or together with BMAL1 ( $n=3$, biological replicates). b Luciferase assay with DISC1 promoter sub-regions: distal part ( -982 to -624 bp relative to TSS), middle part ( -623 to -324 bp relative to TSS), and proximal part ( -323 to +47 bp relative to TSS). Luciferase assay was performed in HEK293 cells overexpressing CLOCK and BMAL1 ( $n=3$, biological replicates). Each group was normalized to the respective control (empty vectors were transfected). $\mathbf{c}$ Luciferase assay with E-box-mutated DISC1 promoter. Luciferase assay was performed in HEK293 cells overexpressing CLOCK and BMAL1 ( $n=3$, biological replicates). Each group was normalized to the respective control (empty vectors were transfected). $\mathbf{d}$ Luciferase assay conducted using the distal part of DISCI promoter with E-box mutations in HEK293 cells overexpressing CLOCK and BMAL1 ( $n=3$, biological replicates). Each group was normalized to the respective control (empty vectors were transfected). e Chromatin immunoprecipitation (ChIP) was performed in HEK293 cells transfected with CLOCK or BMAL1 or both to assess direct binding on E-box sequences of DISC1 promoter ( $n=3$, biological replicates). GFP antibody was utilized to pull down GFP-BMAL1. qPCR was used to quantify the E-box sequences precipitated by GFP-BMAL1. Red arrows indicate estimated primer binding sites for qPCR. For CLOCK and BMAL1 transfection, RFP-CLOCK-myc and GFP-BMAL1 constructs were used for (a-d), and (e). RLA represents relative luciferase activity. Data are means with SEM. ${ }^{*} p \leq 0.05,{ }^{* *} p \leq 0.01,{ }^{* * * *} p \leq 0.0001$, one-way ANOVA and Tukey's post-hoc test for (a-c), (d), and (e).

period of mice, which is represented by the free-running period (Fig. 4d, e). Disc1-LI mice displayed a shorter freerunning period than wild type mice. Furthermore, we examined phase perturbations from external light cues (Supplementary Fig. 3). We gave $30 \mathrm{~min}$ of the light pulse to mice at circadian time $14 \mathrm{~h}$ or $20 \mathrm{~h}$ for phase delay assay or phase advance assay, respectively. Disc1-LI mice exhibited a tendency toward an increase in phase delay and phase advance experiments. However, both did not reach statistical significance.

We also checked the expression of circadian genes at the transcript level. Consistent with the MEFs data, at circadian time $16 \mathrm{~h}$, Disc1-LI mice showed lower expression of Per2, Cry1, and Bmal1 in the hippocampus than 


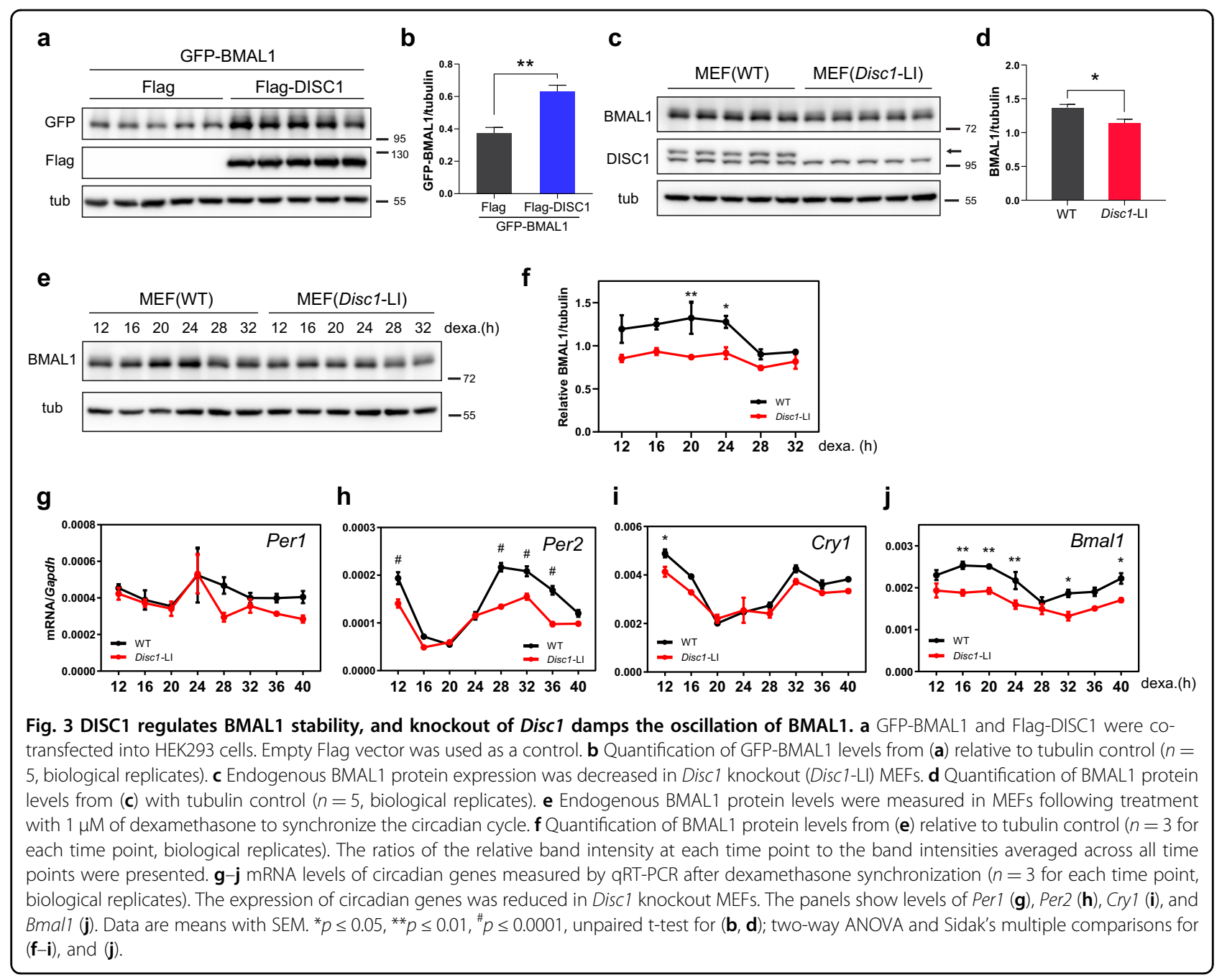

wild type mice (Fig. 4f). These results further demonstrate that DISC1 has a modulatory role for circadian rhythm likely through stabilizing BMAL1, a core component of the molecular clock.

\section{DISC1 regulates the stability of BMAL1 by modulating its ubiquitination through GSK3 $\beta$}

Next, we sought to elucidate how DISC1 regulates the stability of BMAL1. Because DISC1 is known to function as a scaffold protein, we examined the interaction between DISC1 and BMAL1. DISC1 interacted with BMAL1 as shown by co-immunoprecipitation with overexpressed DISC1 and BMAL1 (Fig. 5a). We further confirmed the co-immunoprecipitation in MEFs and mouse brain lysates (Supplementary Fig. 4), suggesting that DISC1 may directly affect BMAL1 function.

Ubiquitination is widely known as a signal for protein degradation ${ }^{52}$. Hence, we conducted ubiquitination assays and observed that ubiquitination of BMAL1 was inhibited upon co-expression of DISC1 (Fig. 5b, c), consistent with the finding that DISC1 increases BMAL1 levels. Conversely, ubiquitination of BMAL1 was enhanced in DISC1 knockdown cells (Fig. 5d, e). Thus, we concluded that DISC1 binds to BMAL1 and inhibits its ubiquitination.

The phosphorylation of glycogen synthase kinase $3 \beta$ (GSK3 $\beta$ ) Ser9 is inhibitory, while Tyr216 phosphorylation enhances its activity $^{53}$. DISC1 can inhibit the Tyr216 phosphorylation (p-Y216) of GSK3 $\beta$ to impede its enzymatic activity ${ }^{30}$. On the other hand, GSK3 $\beta$ regulates multiple circadian proteins ${ }^{54}$, such as CRY2 ${ }^{55,56}$, $\mathrm{CLOCK}^{57}, \mathrm{BMAL1}^{11}$, and REV-ERBa ${ }^{58}$, by modulating their stability ${ }^{10}$. Particularly, BMAL1 phosphorylation by GSK3 $\beta$ promotes its ubiquitination ${ }^{11}$. In addition, as shown in previous reports ${ }^{11,30}$, GSK3 $\beta$ interacts with DISC1 and BMAL1 in co-immunoprecipitation experiments (Supplementary Fig. 4b). Thus, we tested if DISC1 contributes to the regulation of BMAL1 ubiquitination through GSK3 $\beta$. An activity-enhanced form of GSK3 $\beta$ by mutation of Ser9 to Ala increased the ubiquitination of 
a
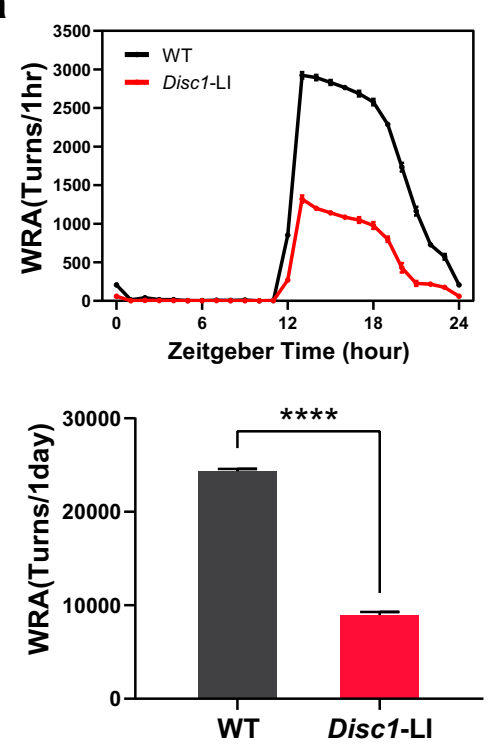

d

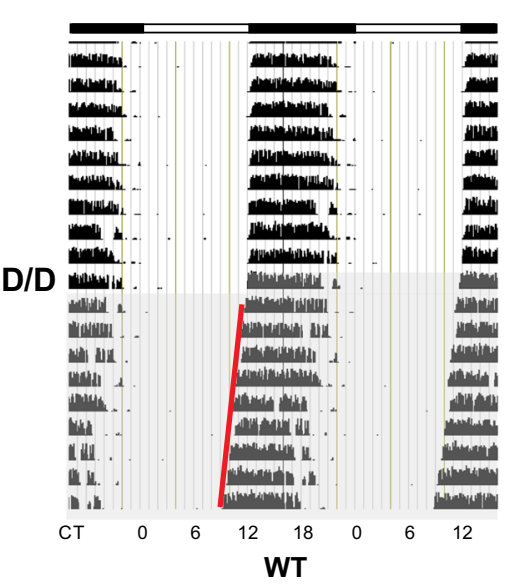

f

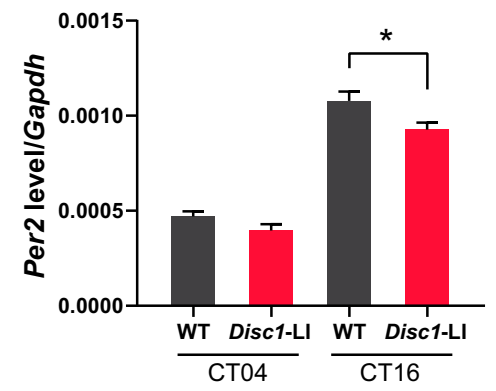

b
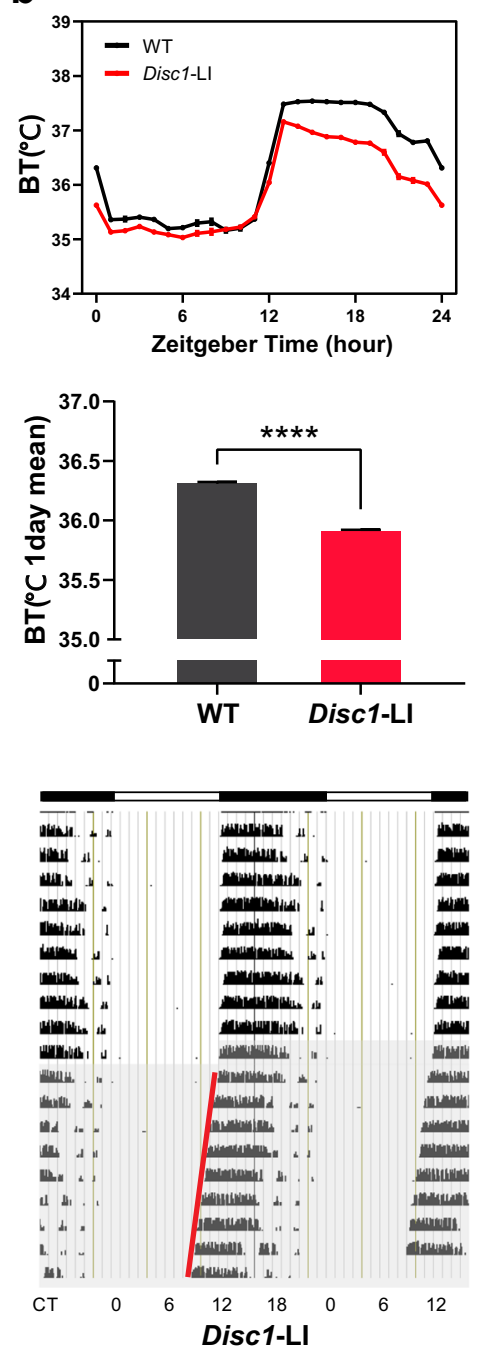

e
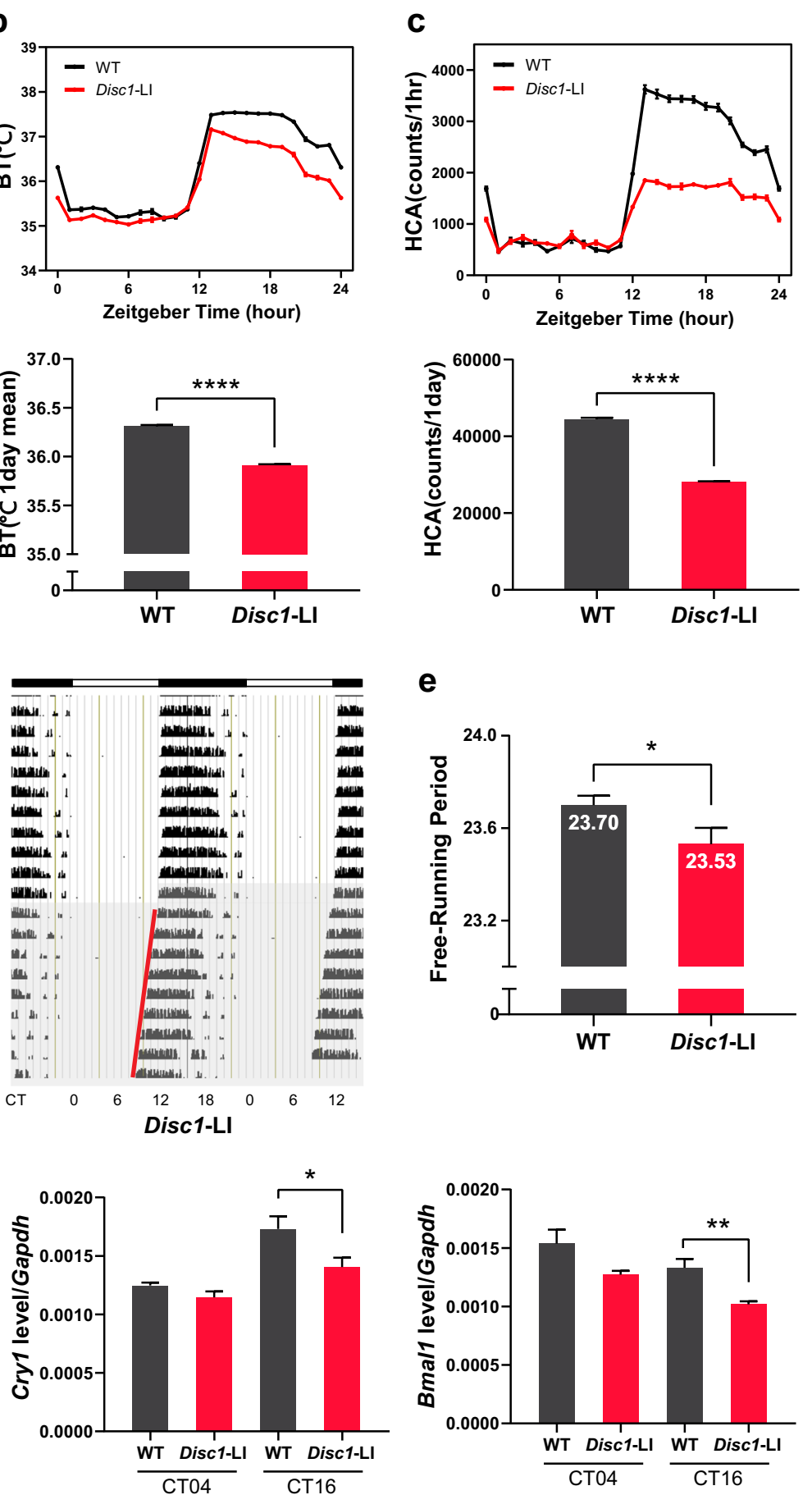

Fig. 4 Disc1 knockout mice exhibit altered circadian behaviors. a-c Overall circadian behaviors during L/D cycle ( $n=10$, biological replicates). Mice were fully entrained to the light/dark (L/D; 12-h/12-h) cycle for at least two weeks. For the next 10 days with the same lighting schedule, light/ dark profiles were generated as described in "Methods". Then, mice were released to a dark/dark (D/D; constant dark) cycle for another 10 days to determine the internal circadian period. a WRA, wheel-running activity; b BT, body temperature; c HCA, home cage activity. Compared to wild type mice, Discl knockout mice (DisC1-LI) showed reduced values during the nighttime. Total measured values per one day were depicted as bar graphs for wheel-running and home cage activities. For body temperature, averaged values were used. $\mathbf{d}$, e Free-running periods were measured for wild type ( $n=16$, biological replicates) and Disc1 knockout ( $n=10$, biological replicates) mice under the D/D cycle. Disc1 knockout mice showed a shorter Free-running period than wild type mice. $\mathbf{f}$ mRNA levels of circadian genes at circadian time of $4 \mathrm{~h}$ (CTO4) and $16 \mathrm{~h}$ (CT16) ( $n=4$ for $4 \mathrm{~h}, n=5$ for $16 \mathrm{~h}$, biological replicates). Hippocampus was dissected from wild type or Disc1 knockout mice. Data are means with SEM. ${ }^{*} p \leq 0.05,{ }^{* *} p \leq 0.01$, ${ }^{* * * *} p \leq 0.0001$, unpaired $t$-test for $(\mathbf{a}),(\mathbf{b}),(\mathbf{e})$ and $(\mathbf{f})$; unpaired $t$-test with Welch's correction for $(\mathbf{c})$. 


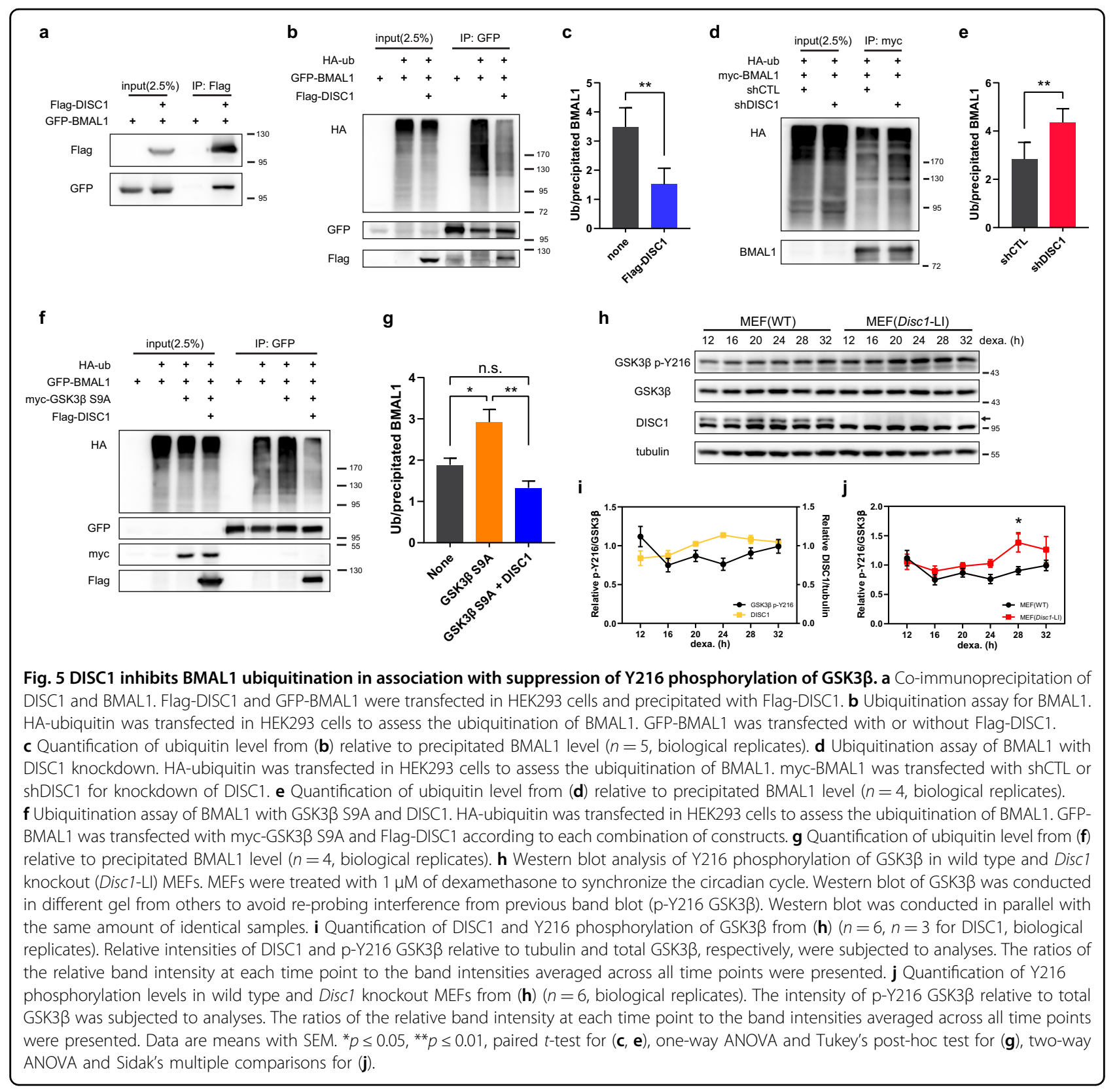

BMAL1 as previously reported (Fig. 5f, g). However, this effect was abolished upon co-expression with DISC1.

To determine the effect of DISC1 on GSK3 $\beta$ over circadian time, we examined the level of p-Y216 in MEFs after dexamethasone synchronization (Fig. 5h). When DISC1 was at its peak, 24 h after synchronization, p-Y216 level was low (Fig. 5i). Subsequently, p-Y216 level gradually increased, while DISC1 level decreased. Moreover, p-Y216 level in Disc1-LI MEFs was increased at $28 \mathrm{~h}$ after dexamethasone treatment compared to wild type (Fig. 5j). Taken together, these results indicate that DISC1 suppresses p-Y216 of GSK3 $\beta$ according to circadian time, thereby enhancing BMAL1 stability.

\section{Discussion}

In this study, we showed that DISC1 has novel functions on the molecular circadian clock, further expanding the repertoire of the diverse roles of DISC1 in various cell types. Interestingly, the functional interaction between DISC1 and the molecular clock is reciprocal; DISC1 modulates the quality of the molecular clock by regulating the GSK3 $\beta$-mediated BMAL1 stability and, at the same time, DISC1 expression itself is under control of CLOCK/ BMAL1, displaying an oscillating expression pattern according to circadian time (Supplementary Fig. 5). The oscillating level of DISC1 is supposed to affect the oscillating level of BMAL1 protein, which will, in turn, affect 
the DISC1 promoter activity. This regulatory loop is achieved by the presence of E-box elements in the DISC1 promoter, which are targets of CLOCK/BMAL1 heterodimer. We identified the two major E-boxes controlled by BMAL1/CLOCK, but more cis-regulatory elements participating in the circadian expression of DISC1 directly or indirectly may still exist. This notion is supported by the observation that the DISC1 promoter activity was not totally abolished by the mutations of both E-box elements. Presumably, as a result, DISC1 deficiency leads to damped expressions of circadian genes. The impacts on the circadian genes were quantitatively differential (Fig. $3 g-j$ ), likely due to the heterogeneity of the compositions and their collective reactivity of the cis-regulatory elements responsible for circadian control $^{59,60}$. Further clarification of this novel modulatory pathway will establish a complete view of the molecular clock.

The peak of DISC1 is similar to the peak of BMAL1, occurring $\sim 20-24 \mathrm{~h}$ after dexamethasone treatment (Figs. 3f and 5i). Moreover, the expression pattern of DISC1 in the brain (Fig. 1b, c, e) is also consistent with the previously reported expression pattern of BMAL1 $1^{9,61,62}$, supporting a protective role of DISC1 for BMAL1. In this regard, the shorter period seen in the Disc1-LI mouse appears counterintuitive, given that mice with low BMAL1 show a more extended per$\operatorname{iod}^{63,64}$ and Bmal1 knockout mice exhibit arrhythmia $^{65,66}$. Notably, however, DISC1 functionally downregulates GSK3 $\beta$ by inhibiting p-Y216. Although there are conflicting results on the relationship between GSK3 $\beta$ and circadian period ${ }^{67,68}$, GSK3 $\beta$ gain-offunction tends to shorten the circadian period ${ }^{69,70}$. Thus, it is possible that the shortened period in Disc1-LI mice is due to the enhanced activity of GSK3 $\beta$, which is consistent with the notion that DISC1 prevents BMAL1 from premature degradation to maintain the robustness of circadian rhythmicity.

We demonstrated that DISC1 expression oscillates according to circadian time. This finding suggests that the previously known functions of DISC1, from embryonic neurodevelopment to adult neurogenesis, may also be regulated by the circadian system. In this respect, the oscillating phosphorylation of Y216, a regulatory hotspot of GSK3 $\beta$ activity controlled by $\operatorname{DISC1}^{30}$, is noteworthy. The phosphorylation of Y216 is critical for neural progenitor proliferation, which may indicate that DISC1-related neurodevelopmental processes, including neuronal signaling, neuronal morphogenesis, and spine regulation ${ }^{17}$, are also under circadian control. Moreover, DISC1 downregulation augments adult neurogenesis in the hippocampus ${ }^{71,72}$ in conjunction with memory formation $^{73}$. Indeed, memory formation and adult neurogenesis are circadian-related phenomena ${ }^{74,75}$. For example, the number of BrdU-positive cells in the hippocampal subgranular zone changes over circadian time, with relatively impaired neurogenesis from late night to early daytime (peak neurogenesis at $15 \mathrm{~h})^{75}$, which corresponds to the level of DISC1 protein expression in the hippocampus shown in this work (Fig. 1e). Therefore, it will be interesting to test whether DISC1 acts as an output pathway that links the molecular clock and neurodevelopmental program.

A natural deletion of Disc1, resulting in truncation after amino acid residue 528, did not alter the sleep phenotypes $^{76}$, which is counterintuitive to our results from Disc1-LI mice. However, it is noteworthy that the truncated DISC1 still retains the region, amino acid residues $195-228$, for the inhibition of GSK3 $\beta$ activity $^{30}$, which is a crucial step of the regulation of BMAL1 stability by DISC1 according to the observations in this study. Its more robust circadian phenotype shown in this study is likely to be a reflection of complete Disc1 loss-of-function. Anyhow, the involvement of DISC1 in the regulation of the molecular circadian clock is intriguing in that sleep and circadian rhythm disruption (SCRD) is a common feature of various psychiatric disorders ${ }^{77}$. Components of molecular clocks were proposed as factors of $\mathrm{SCRD}^{78}$, though the precise molecular mechanisms underlying this phenomenon are still elusive. Although the genetic association of DISC1 with the various psychiatric conditions is still to be further clarified, the identification of DISC1 as a novel regulator of the molecular clock may suggest its role in the interface between some aspects of psychiatric disorders and the circadian system. Notably, rhythmic expression of genes, including PER 2 and $C R Y 2$, is abolished in a group of patients with schizophrenia ${ }^{79}$; similarly, expression of $P E R 2$ and $C R Y 1$ is impaired in some schizophrenia patients ${ }^{80}$. In our study, an interesting correlation was observed in the Disc1 knockout mouse, where the expression of the circadian genes was also diminished. Thus, although the nature of the correlation needs further validation, the roles of DISC1 in the molecular clock may provide a novel mechanistic insight into the molecular links between SCRD and psychiatric disorders.

\section{Acknowledgements \\ This work was supported by the Advanced Research Center Program (Organelle Network Research Center, 2017R1A5A1015366), the Brain Research Program (2017M3C7A1047875) and the Bio \& Medical Technology Development Program (NRF-2020M3E5E2039894) funded by Korean National Research Foundation (S.K.P.).}

\section{Author details}

'Department of Life Sciences, Pohang University of Science and Technology, Pohang, Republic of Korea. ${ }^{2}$ Department of Physiology, College of Medicine, Kyung Hee University, Seoul, Republic of Korea. ${ }^{3}$ Present address: SK biopharmaceuticals Ltd, Seongnam-Si, Republic of Korea. ${ }^{4}$ Present address: Department of Pathology, College of Medicine, Hanyang University, Seoul, Korea 


\section{Author contributions}

S.B.L., Y.K., Y.-U.P., and S.K.P. conceived the study. S.B.L., S.C., and S.K.P. designed experiments. S.B.L., J.P., Y.K., Y.-U.P., T.T.M.N., B.K.S., Y.W., and E.C. performed experiments. S.B.L., B.K.S., Y.W., Y.S., S.C., and S.K.P. analyzed and interpreted data. S.B.L., S.C., and S.K.P. wrote the manuscript.

\section{Funding}

This research was also supported by KBRI basic research program funded by Ministry of Science and ICT (21-BR-03-01).

\section{Conflict of interest}

The authors declare that they have no conflict of interest.

\section{Publisher's note}

Springer Nature remains neutral with regard to jurisdictional claims in published maps and institutional affiliations.

Supplementary information The online version contains supplementary material available at https://doi.org/10.1038/s41398-021-01212-1.

Received: 30 June 2020 Revised: 15 December 2020 Accepted: 7 January 2021

Published online: 04 February 2021

\section{References}

1. Young, M. W. \& Kay, S. A. Time zones: a comparative genetics of circadian clocks. Nat. Rev. Genet. 2, 702-715 (2001).

2. Doherty, C. J. \& Kay, S. A. Circadian control of global gene expression patterns. Annu. Rev. Genet. 44, 419-444 (2010).

3. Bell-Pedersen, D. et al. Circadian rhythms from multiple oscillators: lessons from diverse organisms. Nat. Rev. Genet. 6, 544-556 (2005).

4. Dunlap, J. C. Molecular bases for circadian clocks. Cell 96, 271-290 (1999).

5. Takahashi, J. S. Transcriptional architecture of the mammalian circadian clock. Nat. Rev. Genet. 18, 164-179 (2017).

6. Gekakis, N. et al. Role of the CLOCK protein in the mammalian circadian mechanism. Science 280, 1564-1569 (1998).

7. King, D. P. et al. Positional cloning of the mouse circadian clock gene. Cell $\mathbf{8 9}$, 641-653 (1997).

8. Kume, $K$. et al. mCRY1 and mCRY2 are essential components of the negative limb of the circadian clock feedback loop. Cell 98, 193-205 (1999).

9. Shearman, L. P. et al. Interacting molecular loops in the mammalian circadian clock. Science 288, 1013-1019 (2000).

10. Hirano, A., Fu, Y. H. \& Ptacek, L. J. The intricate dance of post-translational modifications in the rhythm of life. Nat. Struct. Mol. Biol. 23, 1053-1060 (2016).

11. Sahar S., Zocchi L., Kinoshita C., Borrelli E. \& Sassone-Corsi P. Regulation of BMAL1 protein stability and circadian function by GSK3 beta-mediated phosphorylation. PLoS ONE 5, e8561 (2010).

12. Busino, L. et al. SCFFbxl3 controls the oscillation of the circadian clock by directing the degradation of cryptochrome proteins. Science 316, 900-904 (2007).

13. Godinho, S. I. H. et al. The after-hours mutant reveals a role for Fbxl3 in determining mammalian circadian period. Science 316, 897-900 (2007).

14. Zhao, X. et al. Circadian amplitude regulation via FBXW7-targeted REV-ERBa degradation. Cell 165, 1644-1657 (2016).

15. Millar, J. K. et al. Disruption of two novel genes by a translocation cosegregating with schizophrenia. Hum. Mol. Genet. 9, 1415-1423 (2000).

16. Niwa, M. et al. DISC1 a key molecular lead in psychiatry and neurodevelopment: no-more disrupted-in-schizophrenia 1. Mol. Psychiatry 21, 1488-1489 (2016).

17. Brandon, N. J. \& Sawa, A. Linking neurodevelopmental and synaptic theories of mental illness through DISC1. Nat. Rev. Neurosci. 12, 707 (2011).

18. Ishizuka, K., Paek, M., Kamiya, A. \& Sawa, A. A review of disrupted-InSchizophrenia-1 (DISC1): neurodevelopment, cognition, and mental conditions. Biol. Psychiatry 59, 1189-1197 (2006)

19. Wilkinson, B. et al. Endogenous cell type-specific DISC1 interactomes reveal protein networks associated to neurodevelopmental disorders. Biol. Psychiatry 85, 305-316 (2018).
20. Camargo, L. M. et al. Disrupted in Schizophrenia 1 Interactome: evidence for the close connectivity of risk genes and a potential synaptic basis for schizophrenia. Mol. Psychiatry 12, 74 (2006).

21. Sawamura, N. et al. Nuclear DISC1 regulates CRE-mediated gene transcription and sleep homeostasis in the fruit fly. Mol. Psychiatry 13, 1138-1069 (2008).

22. Jaaro-Peled, H. et al. Abnormal wake/sleep pattern in a novel gain-of-function model of DISC1. Neurosci. Res. 112, 63-69 (2016).

23. Wulff, K., Porcheret, K., Cussans, E. \& Foster, R. G. Sleep and circadian rhythm disturbances: multiple genes and multiple phenotypes. Curr. Opin. Genet. Dev. 19, 237-246 (2009).

24. Seshadri, S. et al. Interneuronal DISC1 regulates NRG1-ErbB4 signalling and excitatory-inhibitory synapse formation in the mature cortex. Nature. Communications 6, 10118 (2015).

25. Valenzuela, D. M. et al. High-throughput engineering of the mouse genome coupled with high-resolution expression analysis. Nat. Biotechnol. 21, 652-659 (2003).

26. Longo, P. A., Kavran, J. M., Kim, M.-S. \& Leahy, D. J. Transient mammalian cell transfection with polyethylenimine (PEl). Methods Enzymol. 529, 227-240 (2013).

27. Walker, R. M. et al. The DISC1 promoter: characterization and regulation by FOXP2. Hum. Mol. Genet. 21, 2862-2872 (2012).

28. Rechsteiner, M. \& Rogers, S. W. PEST sequences and regulation by proteolysis. Trends Biochemical Sci. 21, 267-271 (1996).

29. Fukuda, T., Sugita, S. \& Inatome, R. Yanagi S. CAMDI, a novel disrupted in schizophrenia 1 (DISC1)-binding protein, is required for radial migration. The. J. Biol. Chem. 285, 40554-40561 (2010).

30. Mao, Y. et al. Disrupted in schizophrenia 1 regulates neuronal progenitor proliferation via modulation of GSK3 $\beta / \beta$-catenin signaling. Cell 136, 1017-1031 (2009).

31. Kwak, Y. et al. Cyclin-dependent kinase $5(\mathrm{Cdk} 5)$ regulates the function of CLOCK protein by direct phosphorylation. J. Biol. Chem. 288, 36878-36889 (2013).

32. Yoo, S.-H. et al. Period2 3 '-UTR and microRNA-24 regulate circadian rhythms by repressing PERIOD2 protein accumulation. Proc. Natl Acad. Sci. USA 2017, 06611 (2017).

33. Militi, S. et al. Early doors (Edo) mutant mouse reveals the importance of period 2 (PER2) PAS domain structure for circadian pacemaking. Proc. Natl Acad. Sci. USA 113, 2756-2761 (2016).

34. Yin, L., Joshi, S., Wu, N., Tong, X. \& Lazar, M. A. E3 ligases Arf-bp1 and Pam mediate lithium-stimulated degradation of the circadian heme receptor Rev-erba. Proc. Natl Acad. Sci. USA 107, 11614-11619 (2010).

35. Koike, H., Arguello, P. A., Kvajo, M., Karayiorgou, M. \& Gogos, J. A. Disc1 is mutated in the 129S6/SvEv strain and modulates working memory in mice. Proc. Natl Acad. Sci. USA 103, 3693-3697 (2006).

36. Jang, $H$. et al. SREBP1C-CRY1 signalling represses hepatic glucose production by promoting FOXO1 degradation during refeeding. Nat. Commun. 7, 12180 (2016).

37. Lee, S. et al. Valproate alters dopamine signaling in association with induction of Par-4 protein expression. PLOS ONE 7, e45618-e45618 (2012).

38. Lin, S.-T. et al. Nuclear envelope protein MAN1 regulates clock through BMAL1. elife 3, e02981 (2014).

39. Akagi, R. et al. Dysregulated circadian rhythm pathway in human osteoarthritis: NR1D1 and BMAL1 suppression alters TGF- $\beta$ signaling in chondrocytes. Osteoarthr. Cartil. 25, 943-951 (2017).

40. Keller, M. et al. A circadian clock in macrophages controls inflammatory immune responses. Proc. Natl Acad. Sci. USA 106, 21407 (2009).

41. Hirano, A., Braas, D., Fu, Y.-H. \& Ptáček, L. J. FAD Regulates CRYPTOCHROME protein stability and circadian clock in mice. Cell Rep. 19, 255-266 (2017).

42. Son, G. H. et al. Adrenal peripheral clock controls the autonomous circadian rhythm of glucocorticoid by causing rhythmic steroid production. Proc. Natl Acad. Sci. USA 105, 20970 (2008).

43. DeBruyne, J. P. et al. A clock shock: mouse CLOCK is not required for circadian oscillator function. Neuron 50, 465-477 (2006).

44. Ni, Y. et al. The involvement of sympathetic nervous system in essence of chicken-facilitated physiological adaption and circadian resetting. Life Sci. 201, 54-62 (2018).

45. Lee, C. \& Huang, C.-H. LASAGNA-Search: an integrated web tool for transcription factor binding site search and visualization. Biotechniques $\mathbf{5 4}$ 141-153 (2013). 
46. Lee, C. \& Huang, C.-H. LASAGNA-Search 2.0: integrated transcription factor binding site search and visualization in a browser. Bioinformatics 30, 1923-1925 (2014).

47. Kielbasa, S. M., Gonze, D. \& Herzel, H. Measuring similarities between transcription factor binding sites. BMC Bioinforma. 6, 237 (2005).

48. Oishi, K., Shirai, H. \& Ishida, N. Identification of the circadian clock-regulated Ebox element in the mouse plasminogen activator inhibitor-1 gene. J. Thromb. Haemost. 5, 428-431 (2007).

49. Ripperger, J. A. \& Schibler, U. Rhythmic CLOCK-BMAL1 binding to multiple Ebox motifs drives circadian Dbp transcription and chromatin transitions. Nat Genet. 38, 369-374 (2006).

50. Li, R. et al. CLOCK/BMAL1 regulates human nocturnin transcription through binding to the E-box of nocturnin promoter. Mol. Cell. Biochem. 317, 169-177 (2008).

51. Wang, Z., Wu, Y., Li, L. \& Su, X. D. Intermolecular recognition revealed by the complex structure of human CLOCK-BMAL1 basic helix-loop-helix domains with E-box DNA. Cell Res. 23, 213-224 (2013).

52. Clague, M. J. \& Urbé, S. Ubiquitin: same molecule, different degradation pathways. Cell 143, 682-685 (2010).

53. Beurel, E., Grieco, S. F. \& Jope, R. S. Glycogen synthase kinase-3 (GSK3): regulation, actions, and diseases. Pharmacol. Ther. 148, 114-131 (2015).

54. Reischl, S. \& Kramer, A. Kinases and phosphatases in the mammalian circadian clock. FEBS Lett. 585, 1393-1399 (2011).

55. Kurabayashi, N., Hirota, T., Sakai, M., Sanada, K. \& Fukada, Y. DYRK1A and glycogen synthase kinase $3 \beta$, a dual-kinase mechanism directing proteasomal degradation of CRY2 for circadian timekeeping. Mol. Cell. Biol. 30, 1757-1768 (2010).

56. Harada, Y., Sakai, M., Kurabayashi, N., Hirota, T. \& Fukada, Y. Ser-557phosphorylated mCRY2 is degraded upon synergistic phosphorylation by glycogen synthase kinase-3ß. J. Biol. Chem. 280, 31714-31721 (2005).

57. Spengler, M. L., Kuropatwinski, K. K., Schumer, M. \& Antoch, M. A serine cluster mediates BMAL1-dependent CLOCK phosphorylation and degradation. Cell Cycle 8, 4138-4146 (2009).

58. Yin, L., Wang, J., Klein, P. S. \& Lazar, M. A. Nuclear receptor rev-erba is a critical lithium-sensitive component of the circadian clock. Science 311, 1002-1005 (2006).

59. Yamajuku, D. et al. Identification of functional clock-controlled elements involved in differential timing of Per1 and Per2 transcription. Nucleic Acids Res. 38, 7964-7973 (2010).

60. Ukai-Tadenuma, M. et al. Delay in feedback repression by cryptochrome 1 is required for circadian clock function. Cell 144, 268-281 (2011).

61. Chen, L. et al. BMAL1 Disrupted intrinsic diurnal oscillation in rat cerebrovascular contractility of simulated microgravity rats by altering circadian regulation of miR-103/CaV1.2 signal pathway. Int. J. Mol. Sci. 20, 3947 (2019).

62. Barca-Mayo, O. et al. Astrocyte deletion of Bmal1 alters daily locomotor activity and cognitive functions via GABA signalling. Nat. Commun.s 8, 14336 (2017).
63. Landgraf, D. et al. Genetic disruption of circadian rhythms in the suprachiasmatic nucleus causes helplessness, behavioral despair, and anxiety-like behavior in mice. Biol. Psychiatry 80, 827-835 (2016).

64. Mieda, M. et al. Cellular clocks in AVP neurons of the SCN are critical for interneuronal coupling regulating circadian behavior rhythm. Neuron $\mathbf{8 5}$, 1103-1116 (2015).

65. Bunger, M. K. et al. Mop3 is an essential component of the master circadian pacemaker in mammals. Cell 103, 1009-1017 (2000).

66. McDearmon, E. L. et al. Dissecting the functions of the mammalian clock protein BMAL1 by tissue-specific rescue in mice. Science 314, 1304-1308 (2006).

67. Hirota, T. et al. A chemical biology approach reveals period shortening of the mammalian circadian clock by specific inhibition of GSK-3ß. Proc. Natl Acad. Sci. USA 105, 20746-20751 (2008).

68. Besing, R. C. et al. GSK3 activity regulates rhythms in hippocampal clock gene expression and synaptic plasticity. Hippocampus 27, 890-898 (2017).

69. litaka, C., Miyazaki, K., Akaike, T. \& Ishida, N. A role for glycogen synthase kinase$3 \beta$ in the mammalian circadian clock. J. Biol. Chem. 280, 29397-29402 (2005).

70. Martinek, S., Inonog, S., Manoukian, A. S. \& Young, M. W. A role for the segment polarity gene shaggy/GSK-3 in the Drosophila circadian clock. Cell 105 769-779 (2001).

71. Terrillion, C. E. et al. DISC1 in astrocytes influences adult neurogenesis and hippocampus-dependent behaviors in mice. Neuropsychopharmacology $\mathbf{4 2}$, 2242-2251 (2017).

72. Duan, $X$. et al. Disrupted-in-schizophrenia 1 regulates integration of newly generated neurons in the adult brain. Cell 130, 1146-1158 (2007)

73. Kempermann G., Song H. J. \& Gage F. H. Neurogenesis in the adult hippocampus. Cold Spring Harb. Perspect. Biol. 7, a018812 (2015).

74. Gerstner, J. R. \& Yin, J. C. P. Circadian rhythms and memory formation. Nat. Rev. Neurosci. 11, 577-588 (2010).

75. Bouchard-Cannon, P., Mendoza-Viveros, L., Yuen, A., Kærn, M. \& Cheng, H.-Y.M. The circadian molecular clock regulates adult hippocampal neurogenesis by controlling the timing of cell-cycle entry and exit. Cell Rep. 5, 961-973 (2013).

76. Dittrich, L., Petese, A. \& Jackson, W. S. The natural Disc1-deletion present in several inbred mouse strains does not affect sleep. Sci. Rep. 7, 5665 (2017).

77. Wulff, K., Gatti, S., Wettstein, J. G. \& Foster, R. G. Sleep and circadian rhythm disruption in psychiatric and neurodegenerative disease. Nat. Rev. Neurosci. 11 589-599 (2010).

78. Jagannath, A., Taylor, L., Wakaf, Z., Vasudevan, S. R. \& Foster, R. G. The genetics of circadian rhythms, sleep and health. Hum. Mol. Genet. 26 R128-R138 (2017).

79. Seney, M. L. et al. Diurnal rhythms in gene expression in the prefrontal cortex in schizophrenia. Nat. Commun. 10, 3355 (2019).

80. Johansson, A.-S., Owe-Larsson, B., Hetta, J. \& Lundkvist, G. B. Altered circadian clock gene expression in patients with schizophrenia. Schizophr. Res. 174 17-23 (2016). 